\title{
3D Image Reconstruction and Application for Micro-manipulation Systems
}

\author{
Han-Pang Huang ${ }^{*}$ and Fu-Jen $\mathrm{Hsiao}^{+}$ \\ Robotics Laboratory, Department of Mechanical Engineering, National Taiwan University \\ "Professor and correspondence addressee, ${ }^{+}$Graduate student
}

\begin{abstract}
In this paper, a microscopic 3D image is reconstructed for a micro-manipulation system. The $3 D$ image reconstruction is based on a bjnocular stereo vision system. We propose a new method called "support value" to solve correspondence problems Geometric constraints are added to reduce the ambiguities in stereo matching. Left/right consistency check is used to increase the confidence for matching results. Post-processing helps remove the outliers and make original dense disparity map smooth and reliable. The three-dimensional characteristics can be reconstructed by perspective projection. Finally, the 3D image reconstruction algorithm is successfully applied to a micro manipulation system for cell image reconstruction by a simpler and more friendly way.
\end{abstract}

\section{Introduction}

In recent years, due to the growth of micro-mechatronics technology, more emphasis has been placed on the micro manipulation techniques. In order to manipulate micro particles well, there should be suitable sensors to get the three-dimensional range information to help users perform manipulation tasks. Vision is one of the most important sensory modalities used for sensing the operating environment. For example, AFM (atomic force microscope) and SEM (scanning electron microscope) are the most common used visual sensor in micro manipulation. Nevertheless, due to the budget limitation, many research organizations can not afford these expensive instruments. For such reasons, we would like to propose a practical but much cheaper vision system for micro manipulation. The image acquisition instnumem is the most cormmon device: optical microscope. Optical microscope images through two CCD cameras are used to reconstruct the micro level 3D images. The reconstruction results will be presented as virtual reality scene through a virtual graphic engine. The virtual scene will assist a user to get more information and explore more of the original scene.

Among the six steps of stereo vision [1], stereo matching is the most difficult problem. The goal of stereo matching is to solve correspondence problem. The correspondence problem can be stated as follows: for each point in the left image, find the corresponding point in the right image. To determine a conjugate pair, it is necessary to measure the similarity of the points. Thus, it is necessary to locate matchable features before stereo matching. Both edge features and region features have been used in stereo matching.

To solve the correspondence problem, many stereo algorithrns have been proposed for years $[4,5,7,12,18,19]$. Matching techniques can be divided into area-based matching [2][9][20], feature-based matching [16], and a combination of them [3][6]. The area-based techniques attempt to find matches of features presented throughout the image, such as the intensity at each pixel or the correlation of a local window of intensities. The feahure-based techniques attempt to find matches of sparser and more abstract features, rather than matching texture regions in two images.

Other types of stereo matching methods are also proposed for years. Ishikawa and Geiger treated stereo matching problem as a global optimization problem that modeled occlusions, discontinuities and the epipolar line interactions [8]. They mapped the optimization problem to a maximum-flow problem on a directed graph in a novel way and validated their idea to be practical. Zitnick and Kanade presented a cooperative algorithm for stereo matching [21]. The tensor voting enables us to perform complex computations associated with the formulation of the stereo problem at a reasonable cost.

This paper aims to develop a microscopic 3D image for a micro-manipulation system based on a binocular stereo vision system. A new method called "support value" will be proposed to solve correspondence problems. The algorithm will be applied to a micro manipulation systern for cell image reconstruction.

\section{Stereo Matching Algorithm Development}

The sterco matching workflow is sbown schematically in Fig. 1. At first, epipolar lines are aligned to simplify the search range from two-dimension to one-dimension. Matching is driven from left to right, and from right to left, in two separate but identical procedures. The matching candidates are selected by using a proposed criterion called "support value". During the matching process, ordering constraipt and uniqueness assumption are added to reduce the ambiguities and make the matching results more accurate. The determination of correspondence is based on not only the conventional criterion "cross-correlation" but also the proposed "support value".

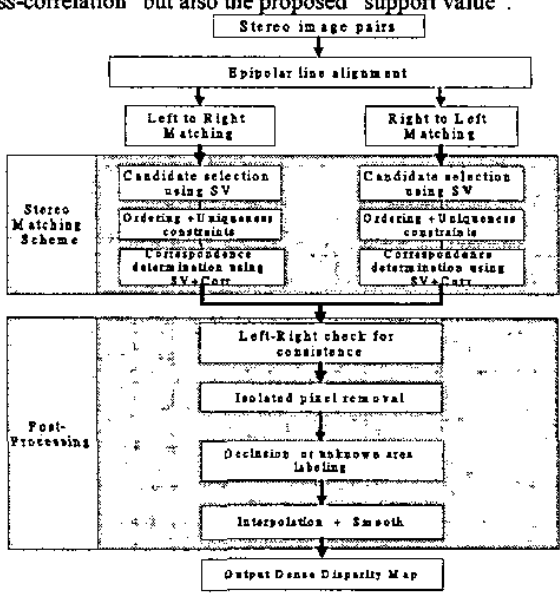

Fig. 1 Flowchart of stereo matching algorithm

2.1 Support Value

The original ideas of support value come from the structures similarity of stereo image pairs. It is deeply believed that structures like comers, features or other special structures of the left eye image are similar to those of the right eye image. The structure mainly depends on its neighborhood pixels. There should exist close neighborhood correlation of the structure. In other words, while doing stereo matching tasks, its neighborhood will contribute some "cues" for the correct match. Therefore, we define a value called "Support Value" and a complete criterion in aid of stereo matching to realize the above idea.

Support value calculation procedures are as follows:

1. Calculate local mean values of each local support.

$$
m_{l}=\frac{1}{N} \sum_{i} \sum_{j} f_{i}(i, j) ; m_{2}=\frac{1}{N} \sum_{i} \sum_{i} f_{l}(i, j)
$$

2. Threshold the local support to obtain the binary structure.

if $f_{1}(i, j) \geq m_{1}, \quad S_{1}(i, j)=1 \quad$ else $S_{1}(i, j)=0$;

if $f_{r}(i, j) \geq m_{r}, \quad S_{r}(i, j)=1$ else $S_{r}(i, j)=0$

3. Compare the binary structure and add scores to support value. For each $S_{1}$ and $S_{\text {r }}$ element,

if $S_{l}(i, j)=S_{r}(i, j), S V=S V+W(i, j)$

4. Use support value for candidate selection.

Support value is used to select the possible candidates.

5. Determine corresponding point using cross-correlation and support value.

The candidate whose support value is maximum can be 
considered as a good match. However, correspondence determination using only single criterion may lead to erroneous results. Here we adopt multiple criteria to avoid this situation. The support value is used to select the matching candidate first, and then the combination of correlation and support value decides which one is the corresponding point. The correspondence determination flowchart is described in Fig. 2.

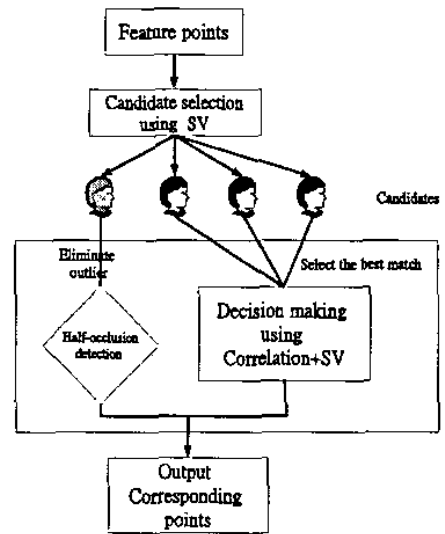

Fig. 2 Correspondence determination flowchart

The value of support value depends on the weighting matrix. The coefficient assignment will have a great influence on the matching results. The common used weighting matrices are listed in Fig. 3. In general, equally weighted matrix treats each neighbor in a fair state. Decreasing-order weighted matrix treats each neighbor in another way. The window size of the support value depends on the texture of matching areas.
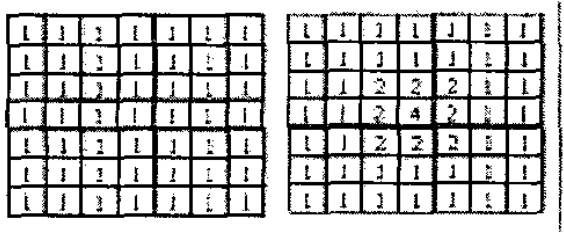

Fig. 3 a) Equally weighted matrix, b) decrease-order weighted matrix

\subsection{Constraints}

The correspondence problem is ambiguous. Given a point $\mathrm{ml}$ in the left image, it may correspond to any point $\mathrm{m} 2$ in the right image. To solve the ambiguity, some constraints must be added to reduce the number of potential matches for any given point $\mathrm{ml}$.

The constraints used in the matching process of this paper are listed below:

a). Epipolar constrain

b). Disparity range constraint [10]

c). Uniqueness constraint

d). Continuity constraint [15]

e). Ordering constraint

2.3 Consistency Check

After the process of left-to-right matching and right-to-lef matching, a mechanism needs to be established to validate the matching results. The most commonly used mechanism is leftright consistency check or bi-directional validation. In Fig. 4, it first compares the left-to-right results with the right-to-left results, check their agreements, and then consider them as the correct matches. At first, this agreement on matched points must be exact, but it is often relaxed to allow 2 to 4-pixel disagreement so that the disparity surface is allowed to incline rather than perpendicular to the camera axis.

Although left/right consistency check takes twice computation time, it indeed provides confidence for the disparity estimation and three-dimensional coordinates calculation. In this paper, we adopt this strategy with its relaxation to validate the matching results.

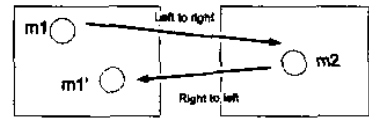

Falso match

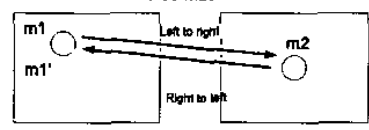

Correct match

Fig. 4 Bi-directional validation (left/right consistency check)

\subsection{Occlusion Detection}

Binocular half-occlusion points are those that are visible in one of the two views but occluded in another view. Due to their importance in stereo matching and subsequent interpretation tasks, a number of approaches have been developed for dealing with occlusion points. Fig. 5 shows a simple example of binocular imaging system with occlusion situation. In such a situation, since there exist depth discontinuities, the disparity estimation within these segments may lead to enoneous results.

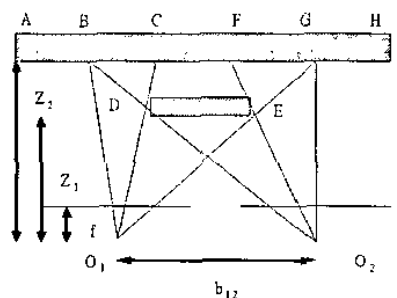

Fig. 5 Occlusion situation in binocular system

According to certain geometric assumptions and constraints, occlusion detection approaches can be roughly divided into five approaches [5]. One of these approaches is Left/Right Checking (LRC). Those points with inconsistent left/right checking are considered the false matches and occlusion points. They need to be excluded in the subsequent processes of disparity estimation. The disparities of occlusion areas are interpolated by neighboring correct matches.

\subsection{Disparity Estimation and 3D Reconstruction}

\subsubsection{Disparity Estimation}

After validation, the corresponding pairs are considered as correct matches. In order to estimate depth, the disparity needs to be calculated first. Given a corresponding pair $\left(x_{i}, y_{1}\right)$ and $\left(x_{r}, y_{r}\right), y_{1}$ must equal to $y_{\mathrm{r}}$ due to the epipolar constraint. The left-based disparity or parallax is defined as $x_{t}-x_{r}$, while the right-based disparity is defined as $x_{r}-x_{1}$. The parallax is the reason why human can perceive depth. Through the brain activation, parallax will be translated into distance sense for human to tell which is far and which is near.

After calculating all corresponding pairs, a dense disparity map can be obtained except for the occlusion areas. A pseudo-color map represents the disparity image. In a disparity map, there are still some areas with unknown disparities. These areas are occlusion areas. The disparities of occlusion areas need to be interpolated to recover the depth. Based on the disparity gradient limit, the disparities of occlusion areas must lie between the disparities of neighborhood areas. Here we adopt linear interpolation to recover the lost disparities.

\subsubsection{D Reconstruction}

The geometry of binocular imaging system is shown in Fig. 6 . The setup is two identical cameras separated in the $\mathrm{x}$-direction by a baseline distance $b$. The image planes are coplanar in this case. For the model shown in Fig. 6, every feature in one image will lie on the same row in the second image. In practice, there may be a vertical disparity due to the misregistration of the epipolar lines. Many formulations of binocular stereo algorithms assume zero vertical disparity. This is also adopted in this paper. 


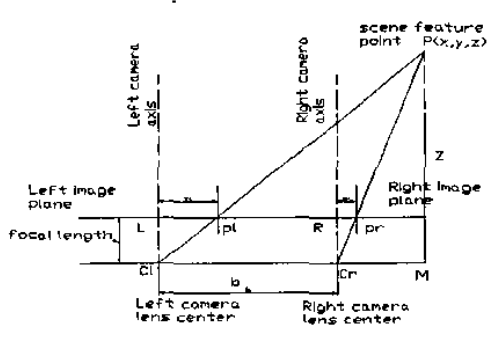

Fig. 6 Binocular imaging system setup

In Fig. 6, the feature point $P$ is observed at points $\mathrm{pl}$ and $\mathrm{pr}$ in the left and right image planes, respectively. In general, we assume that the origin of the world coordinate system coincides with the midpoint of the left camera center and the right camera center. Therefore the left camera center is located at $\left[\begin{array}{llll}-\frac{b}{2} & 0 & 0\end{array}\right]^{r}$, and the right camera center is located at $\left[\begin{array}{lll}\frac{b}{2} & 0 & 0\end{array}\right]^{r}$.

Comparing the similar triangles $\mathrm{PMC}_{\mathrm{b}}, \mathrm{p}_{\mathrm{L}} \mathrm{LC}_{\mathrm{l}}$ and $\mathrm{PMC}$ and $\mathrm{p}, \mathrm{RCr}$ we can obtain

$$
\frac{x+\frac{b}{2}}{z}=\frac{x_{i}}{f} ; \frac{x-\frac{b}{2}}{z}=\frac{x_{r}}{f}
$$

Combining the above two equations, we get

$$
z=\frac{b^{*} f}{\left(x_{1}-x_{r}\right)}=\frac{b^{*} f}{D}, \quad D: \text { disparity } b: \text { baseline } f: \text { focal length }
$$

Thus, the depth at various scene points may be recovered by knowing the disparity of corresponding feature points.

From the above discussion, if a conjugate pair can be determined, the depth in world coordinate system $Z$ can be easily derived. Knowing the depth $Z$, the $\mathrm{X}, \mathrm{Y}$ coordinate in world coordinate system can be derived using perspective projection equations as

$$
\begin{aligned}
& X=\frac{b^{*}\left(x_{i}+x_{r}\right)}{2^{*}\left(x_{i}-x_{r}\right)} \\
& Y=\frac{b^{*}\left(y_{1}+y_{r}\right)}{2^{*}\left(x_{t}-x_{r}\right)}=\frac{b^{*} y}{x_{l}-x_{r}} \quad\left(y_{l}=y_{r}=y\right)
\end{aligned}
$$

\section{Microscopic 3D Image Reconstruction}

\subsection{Introduction to Micro-manipulation Systems}

The micro-manipulation system shown in Fig. 7 and Fig. 8 consists of a stereo microscope, two 3-DOF robotic manipulators, a micro-positioning platform, a micro-injector, and a central computer. It is desired to construct a stereo vision system suitable for micro manipulation. Microscopic images captured by the left and right CCD cameras are used for stereo matching task. The algorithm mentioned in section 2 is used to estimate the depth in the three-dimensional scene. With accurate depth estimation, the relative positions of the manipulator and objects can be easily obtained. The depth information will be sent to the central computer for path planning. The manipulator needs obstacle avoidance paths to reach the destination in the process of transportation. The paths of the manipulators are planned beforehand via a fuzzy-based obstacle avoidance algorithm [13]. The central computer will send instructions to the manipulator and the piezo-driven precision positioning stage with a micro injector. The mechanism and controller of the precision positioning stage are designed by Lan [11]. The robotic manipulator and the micro positioning stage are in charge of translating or assembling micro particles, even injecting particles into cells. The entire system is designed for automatic bio-cell injection. The system can also be acted as an "automated precise micro-assembly system" Fig. 7 and Fig. 8 show the appearance and the architecture of the entire system, respectively.

\subsection{Cell lmage Segmentation}

The textureless background will cause difficulties while doing stereo matching tasks. Therefore, image segmentation needs to be done first to determine the cell positions and contours. Instead of matching the entire image, only those parts having cells proceed stereo matching task. The background will be discarded in stereo matching. The cell image segmentation flow is shown in Fig. 9. The variance images are calculated first for later binarization. An automatic threshold selection for image binarization is adopted for separating the background and cells. The connected components labeling is used to group pixels into regions or objects. Finally, a boundary extraction algorithm is developed to determine the positions and contours of cells.

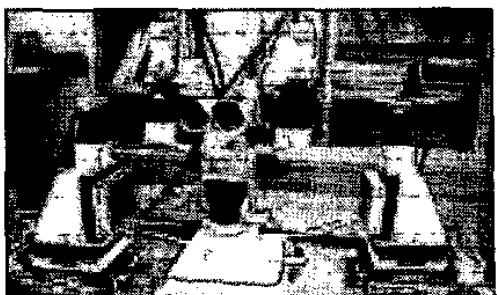

Fig. 7 Appearance of micro manipulation system

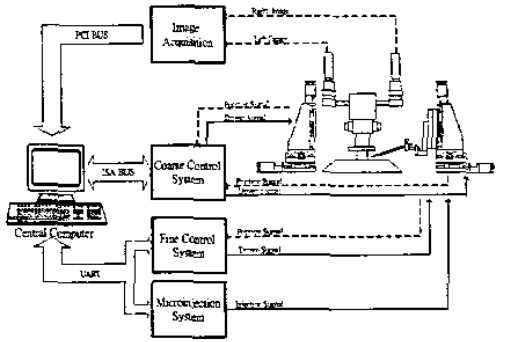

Fig. 8 Architecture of micro manipulation system

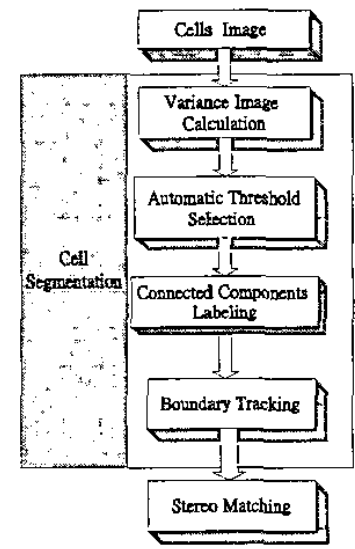

Fig. 9 Cell segmentation flow

\subsubsection{Variance Image Calculation}

Without loss of generality, there are much difference in image intensity between cells and background areas. Therefore, the variances near cell contour are always large. This property can be utilized to detect the rough position of cells. A variance image, which calculates gray level variance of neighborhood in a specific range, is a normalized gray level variance map that all variance values fall between 0 and 255 . The definition of variance is

$$
\sigma^{2}(i, j)=\frac{1}{(2 \times M+1)^{2}} \sum_{,=-M-M}^{i+M} \sum_{i=j-M}^{j+M}[I(s, t)-u(i, j)]^{2}
$$

where $I(i, j)$ means the gray level intensity in image coordinate $(i, j)$ $2 \mathrm{M}+1$ is the range of variance window, and $u(i, j)$ is the average gray level intensity in variance window, which is defined as

$$
u(i, j)=\frac{1}{(2 \times M+1)} \sum_{s=i-M}^{i+M} \sum_{i=j-M}^{i+M} I(s, t)
$$




\subsubsection{Automatic Threshold Selection}

Using variance image, the rough position of cells can be recognized, but still imprecise enough. We adopt a nonparametric and automatic threshold selection for separating objects from background. An optimal threshold is selected by the discriminant criterion, namely, to maximize the separability of the resultant classes in gray levels. The algorithm is proposed by Ostu [17].

Once the optimal threshold is selected, objects can be separated from background. Thus, the positions of objects can be determined automatically.

\subsubsection{Connected Components Labeling}

After separating background from objects successfilly, connected components labeling is used to count the number of object. Connected components labeling is an image processing technique that can make a unit change from pixels to regions or segments. It scans an image and groups its pixels into connected components based on pixel connectivity. Namely, all pixels in a connected component share similar pixel intensity values and are connected to each other in the same way. Once all groups have been determined, each pixel is labeled with a color according to the component it was assigned.

\subsubsection{Boundary Extraction}

Given each object a unique label, the initial position of the object can be estimated by calculating its centroid, but the exact boundary is still unknown. In order to obtain a complete contour, a boundary extraction algorithm proposed by Liu [14] is used to extract the exact boundary. The algorithm modifies chain code method with specific searching orders. The traditional chain code always search for the same direction, while this algorithm searches the next boundary pixel in certain directions.

Direction is defined and given by

direction $[m]=(x[m], y[m])-(x[m-1], y[m-1])$

where $m$ is the index of the current point and $m-1$ is the index of the previous point. Fig. 10 shows the definition of the direction for boundary extraction and the corresponding eight-neighbor pixels.

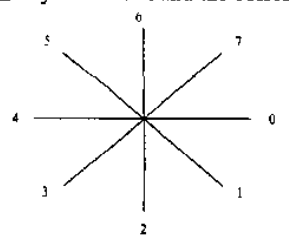

\begin{tabular}{|c|c|c|}
\hline P5 & P6 & P7 \\
\hline P4 & $\times[m], 9[m]$ & P0 \\
\hline P3 & P2 & P1 \\
\hline
\end{tabular}

Fig. 10 Definition of direction and corresponding eight-neighbor pixels

In a regular chain-code method, the search direction of the next pixel always begins at direction 0 and follows clockwise. However, the searching direction of the boundary following algorithm is according to the following rules:

$$
\begin{aligned}
& \text { Rule } i: \text { If direction is }(i-1) \text {, then the next point is } P_{i+5} \text {, for } 0<i<3 \\
& \text { If direction is }(i-1) \text {, then the next point is } P_{i-3} \text {, for } 3<i<8
\end{aligned}
$$

Once the starting point is searched again, the boundary extraction procedure is accomplished.

\subsection{D Reconstruction of Microscopic Cell Images}

The procedure of $3 \mathrm{D}$ reconstruction of cell images is shown in Fig. 11. The stereo cell image pairs will be preprocessed for epipolar line atignment or noise removal. After successfully segmenting the cell part from the background, the corresponding relation of each blob and its boundary information will be recorded for next process. The stereo matching scheme will continue until all cell parts are matched. Post-processing, such as left/right consistency check, isolated pixel removal, and interpolation, will be done after stereo matching scheme. Finally, the dense disparity map and $3 \mathrm{D}$ cell images will be displayed on the monitoring system.

The actual three-dimensional coordinates in world coordinate system addressed in the previous section needs to be modified since the microscope / camera adapter combination is more complex than a single camera. $z=\frac{f \times B}{D}$ is modified by substituting the effective focal length of the microscope / camera adapter combination $f_{\text {t }}$ for the focal length of a single camera. The effective focal length is estimated in the calibration process.

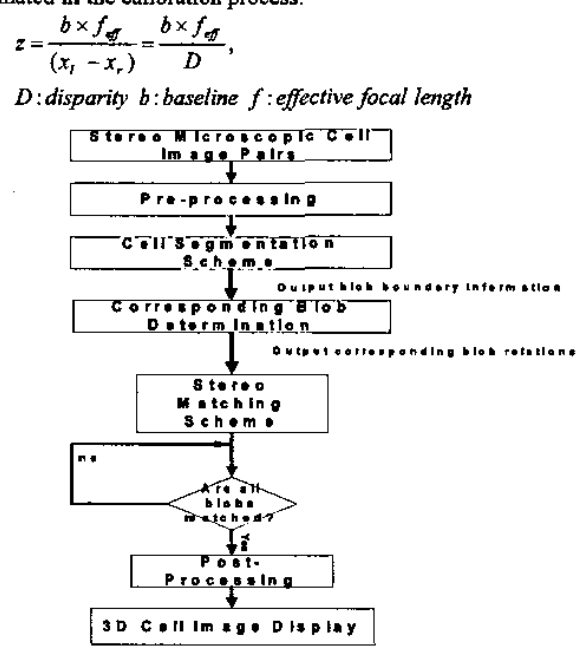

Fig. 11 Flowchart of 3D reconstruction of cell images

\section{Experimental Results}

\subsection{Traditional Stereo Images Test}

The stereo image pairs that have been rectified in advance are obtained from CMU image database [22]. The main hardware includes a personal computer with Intel Pentium $4.18 \mathrm{GHz}, 256 \mathrm{MB}$ DDR RAM, and the optical system of the micro manipulation system.

"Pentagon" is still used for 3D image reconstruction. Due to the disparity range constraint mentioned in section 2.2 , a proper disparity search range must be decided. The technique we adopt is to choose the disparity search range that maximizes the bi-directional validation correct ratio. As shown in Fig. 12, the disparity search range 10 obtains a maximum correct ratio $(95.19 \%)$, thus we use $(10,-10)$ for search in the object window. In other words, if the point is $P(x, y)$ in the reference image, we will search from $P^{\prime}(x-10, y)$ to $P^{\prime}(x+10, y)$ in the object image to determine the best match since it is an one-dimensional search.

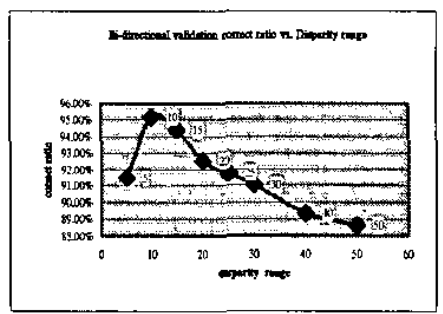

Fig. 12 Disparity range vs. correct ratio

The stereo matching parameters are listed below: support value window $13 \times 13$, support value equally weighted weighting matrix disparity search range 10 -10. The left-to-right and right-to-left matching results will be validated by left/right consistency check. Only the consistent ones are considered as correct matches. Fig. 13 is the result of bi-directional validation. It shows that most matches $(86.42 \%)$ are consistent without relaxation, even the case that the error rate is greater than one pixel is only $1.23 \%$. It provides more confidence for later disparity estimation.

After bi-directional validation, the maximum and minimum disparity is $[10,-9]$, and this is very close to the ground truth disparity range $[8,-8]$ recorded in the document [7]. The occlusion areas in the left and right image are shown in Fig. 14. 


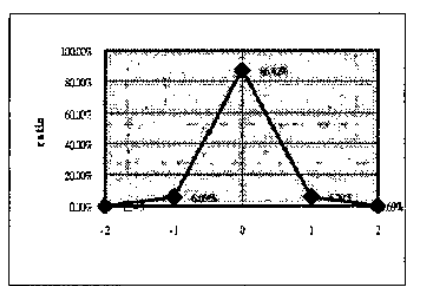

Fig. 13 Validation result analysis
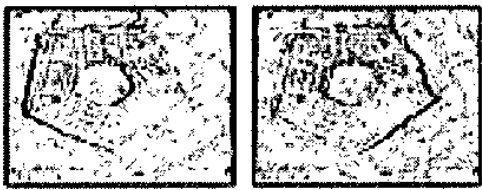

Fig. 14 a). Right occlusion area, b). Left occlusion area

The dense disparity map will be sequentially processed by isolated pixel removal, smoothing, and interpolation to make up the disparities of the occlusion area. The final disparity map in gray level and pseudo color representation is shown in Fig. 15. The brighter color means the larger disparity value, while the darker color means the smaller disparity value. Thus, we can tell the pentagon is higher than its surroundings. Table 1 is the comparison of different stereo matching methods, and the results show that the proposed method can obtain better performance than other methods.
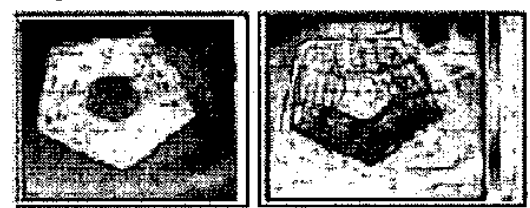

Fig. 15 (a) Disparity map after post-processing, (b) pseudo color disparity map

Table 1 Comparison of pentagon stereo (a) Cox's method [4], (b)

Rov's method [18], (c). Sun's method 02 [19], (d) Our method

(a) (b)

(c) (d)

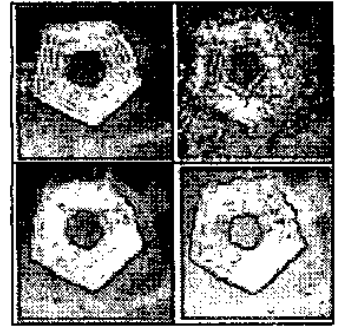

The three-dimensional disparity map is also shown in Fig. 16. It reveals fine structures of pentagon, even the small details. However, the three-dimensional scene can not be teconstructed due to lacking of image acquisition device information, such as focal length, baseline information.
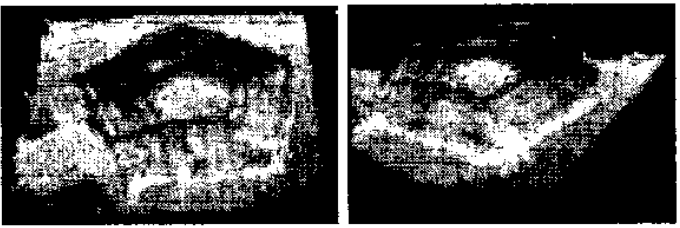

Fig. 16 3D image reconstruction result (pentagon)

\subsection{Microscopic Images Test}

The optical system of the micro manipulation system includes a stereo light microscope, two CCD cameras, two frame grabbers, and a video distributed amplifier. The stereo image pairs are acquired using two CCD cameras attached to the stereo light microscope with standard optical adapters. Thus, the microscope / camera adapter combination is more complex than the traditional binocular stereo system. The stereo light mictoscope we use is Olympus SZX 12, and the CCD cameras are Mintron 8065CB.

The cells we use for microscopic image reconstruction are osteoblasts and osteoclasts of mice. The osteoblasts are cells which aid the growth and development of bones, while the osteoclasts are cells which actively reabsorb old or fatigued bone so that new bone may be replaced by osteoblast cells. Osteoblast cells will cluster together and finally produce osteoclast cells. Osteoclast cells are usually larger than the osteoblast cells several times and have diameter from $20 \mu \mathrm{m}$ to $100 \mu \mathrm{m}$. The image segmentation algorithm will be tested using osteoblast and osteoclast microscopic images.

Osteoblast cells are used to test the algorithms. Fig.17 and Fig.18 show the histograms of the left variance images and the left eye image, respectively. The red arrow indicates the suitable threshold calculated by the algorithm mentioned in section 3.2.2. Therefore, the boundary extraction algorithrn can be applied to the cell parts. Fig. 19 illustrates the good performance of tracking boundaries of cells.

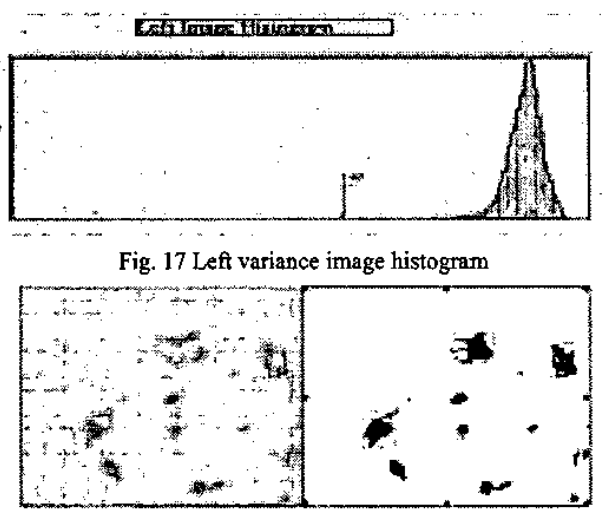

Fig. 18 Left eye image of osteoblast cells a). Original image b). binarized image

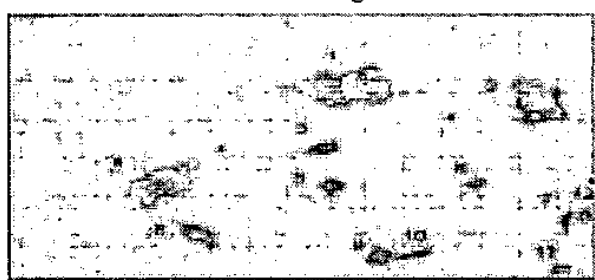

Fig. 19 Boundary extraction result

Various boundary extraction results are listed in Table 2. The results show the robustness and fitness of boundary extraction algorithm to different images.

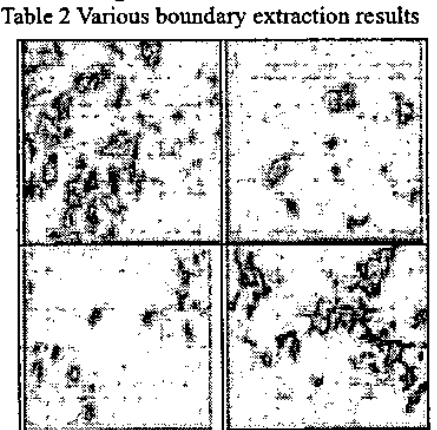

Upon knowing the boundary information of each cell, the stereo matching procedure can be proceeded. Bi-directional validation will 
validate the left-based and right-based matching results. After post-processing, the pseudo-color disparity map is shown in Fig. . Fig. 21 is the front view of $3 \mathrm{D}$ image reconstruction result. The fine structures of cells are also shown in Fig. 22.

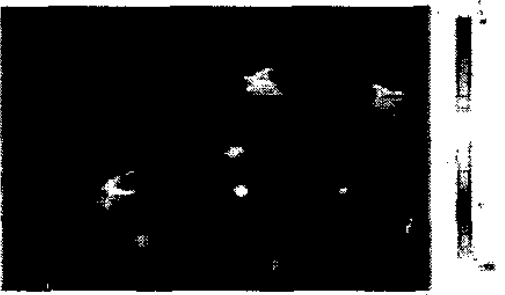

Fig. 20 Pseudo-color disparity map

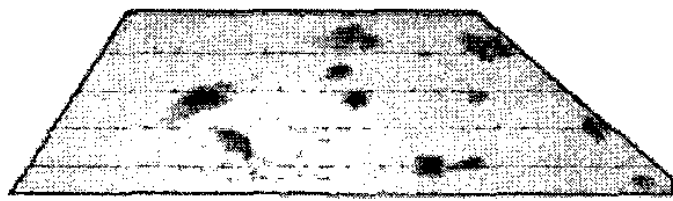

Fig. 21 Front view of 3D image reconstruction results

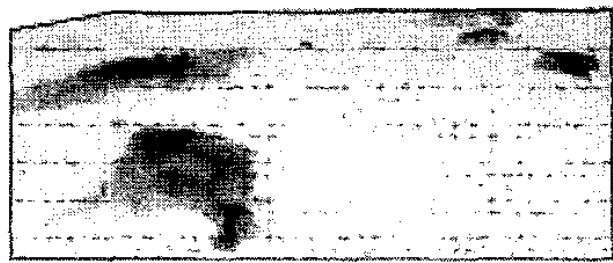

Fig. 22 Fine structures of cells

\section{Conclusions}

In order to construct a stereo vision system for micro manipulation, a complete and practical procedure is constructed to reach the goal. It can be divided into two main tasks. One is stereo matching methodology, the other is cell image segmentation technique.

The experiments obtain successful 3D image reconstruction results. It validates the feasibility of combining the image segmentation techniques with stereo matching methodology to do 3D reconstruction of microscopic cell images. The estimated position and contour information will help manipulation process a lot. More complex manipulation processes such as transportation, assembly, or injection can be performed with the auxiliary vision information.

\section{References}

[1] S. Bamard and M. Fischler, "Computational Stereo," $A C M$ Computing Surveys, Vol. 14, pp. 553-572, 1982.

[2] W. Cheng, J. Liu, and J. Zhang, "A Binocular Computer Vision System for Aerial Image Pairs," Praceedings of .Intemational Conference on Signal Processing, Vol. 2, pp. 954-957, 1996

[3] S. D. Cochran, and G Medioni, "3-D surface description from binocular stereo," IEEE Transactions on Pattern Analysis and Machine Intelligence, Vol. 14, Issue 10, pp. 981-994, 1992.

[4] I. J. Cox, S.L. Hingorani, S.B. Rao, and B.M. Maggs, "A Maximum Likelihood Stereo Algorithm," Computer Vision and Image Understanding, Vol. 63, No. 3, pp. 542-567, 1996.

[5] G. Egnal and R. P. Wilds, "Detecting Binocular Half-occlusions: Empirical Comparisions of Five Approaches," IEEE Transactions on Pattern Analysis and Machine Intelligence, Vol. 24, Issue 8, pp. 1127-1133, 2002.

[6] E. E. Hemayed, M. S. Brown, A. A. Farag, and W. B. Seales, "Cooperative Stereo: Combining Edge- and Area-based
Stereo," Proceedings of IEEE Conference on Aerospac, Vol. 3 , pp. 421-428, 1999.

[7] Y. C. Hsieh, D. M. McKeown, and F. P. Perlant, "Performance Evaluation of Scene Registration and Stereo Matching for Cartographic Feature Extraction," IEEE Transactions on Pattern Analysis and Machine Intelligence, Vol. 14, Issue 2, pp 214-238, 1992.

[8] H. Ishikawa, "Multi-scale Feature Selection in Stereo," Proceedings of IEEE Society Conference on Computer Vision and Pattern Recognition, Vol. 1, pp. 1132-1137, 1999.

[9] T. Kanade and M. Okutomi, "A Stereo Matching Algorithm with an Adaptive Window: Theory and Experiment," IEEE Transactions on Pattern Analysis and Machine Intelligence, Vol. 16, Issue 9, pp. 920-932, 1994.

[10] D. Kuan, "Constraints and Consistency in Stereo Matching," Proceedings of IEEE Conference on Acoustics, Speech, and Signal Processing, Vol. 11, pp. 2079-2082, 1986.

[11] W. H, Lan, "Development of the Micro-Positioning Stage and Precision Motion Controller of the Micromanipulation System," Master thesis, Department of Mechanical Engineering, National Taiwan University, 2003.

[12] M. S. Lee, G Medioni, and P. Mordohai, "Inference of Segmented Overlapping Surfaces from Binocular Stereo," IEEE Transactions on Pattern Recognition and Machine Intelligence, Vol. 24, Issue 6, pp. 824-837, 2002.

[13] C. F. Lin, "Application of Plan Planning to Micro Manipulation System," Master thesis, Department of Mechanical Engineering, National Taiwan University, 2003.

[14] Y. H. Liu, "Feature Analysis and Classifier Design and Thei Applications to Pattern Recognition and Data Mining," Ph.D. Dissertation, Department of Mechanical Engineering, National Taiwan University, 2003.

[15] D. Marr, Vision: A Computational Investigation into the Human Representation and Processing of Visual Information, W. H. Freeman and Company, New York, 1982

[16] P. Moallem and K. Faez, "Fast Edge-based Stereo Matching Algorithm Based on Search Space Reduction," Proceedings of IEEE Workshop on Neural Nenworks for Signal Processing, pp. $587.596,2002$

[17] N. Otsu, "A Threshold Selection Method from Gray-level Histograms," IEEE Transactions on System, Man, and Cybernetics, Vol. 9, No. 1, pp. 62-66, 1979.

[18] S. Roy, "Stereo without epipolar lines: A maximum-flow formulation," International Joumal of Computer Vision, Vol. 34 , No. 2/3, pp. 141-161, 1999.

[19] C. Sun, "Fast Stereo Matching Using Rectangular Subregioning and 3D Maximum-surface Techniques," International Journal of Computer Vision, Vol. 47, pp. 99-117, 2002.

[20] G Thorpe and D. Fraser, "Problems in Area-based Image Registration for Stereo," Proceedings of IEEE Conference on Speech and Image Technologies for Computing and Telecommunications, Vol. 1, pp. 347-352, 1997.

[21] C. Zitnick and T. Kanade, "A Cooperative Algorithm for Stereo Matching and Occlusion Detection," IEEE Transactions on Pattern Analysis and Machine Intelligence, Vol. 22, Issue 7, pp $675-684,2000$

[22]

http:/www.microscopyu.com/articles/stereomicroscopy/stereo intro.html 